\title{
Biomimetic Impedance Control of an EMG-Based Robotic Hand
}

\author{
Toshio Tsuji ${ }^{1}$, Keisuke Shima ${ }^{1}$, Nan $\mathrm{Bu}^{2}$ and Osamu Fukuda ${ }^{2}$ \\ 1: Graduate School of Engineering, Hiroshima University, \\ 2: Measurement Solution Research Center, National Institute of \\ Advanced Industrial Science and Technology \\ Japan
}

\section{Introduction}

The number of extremity amputations resulting from workplace mishaps, traffic accidents and other incidents has shown an increasing trend over time, although the importance of safety management and the prevention of such accidents is fully recognized. Since precise and complex motion may be very difficult in the daily activities of amputees, the development of prosthetic systems is necessary to support their lives and enable social integration. In particular, there is a mandatory requirement for the development of externally powered prosthetic hands with a natural feeling of control, since the role played by this part of the body is very important. However, the control of such hands is problematic, and they must be carefully designed in line with the amputee's remaining functions.

Many researchers have designed prosthetic limbs for amputees since the concept was proposed by N. Wiener in Cybernetics [1]. In previous research, electromyograms (EMGs) have been widely used as an interface tool for prosthetic hands because EMG signals contain information about the operator's intended motion [2] - [8]. For example, an EMGprosthetic hand made in the USSR [2], the Waseda hand developed by Kato et al. [3], the Boston arm by MIT [4] and the Utha artificial arm by Jacobson et al. [5] were all pioneering steps in the field. Since EMG signals also include information on the force level and mechanical impedance properties of limb motion, Akazawa et al. [6] estimated the force of flexors and extensors from these signals and proposed a scheme to use them in controlling a prosthetic hand. Abul-haj and Hogan [8] also proposed prosthetic control based on an impedance model and analyzed its control characteristics.

Most previous research, however, dealt only with on/off control for prosthetic arms depending on the results of EMG pattern discrimination [2], [3], [7], or controlled only a particular joint depending on the torque estimated from EMG signals [4], [5], [6], [8]. Multijoint control of prosthetic arms considering the variable viscoelasticity of flexors and extensors has not yet been realized.

This chapter introduces a biomimetic control for an externally powered multi-joint prosthetic hand that considers the muscular contraction levels of flexors and extensors using 
neural networks. The method can express the difference between internal and external forces arising from the flexors and extensors, and all joints can be controlled as intended by the amputee. A natural feeling of control similar to that of the human arm can also be expected, since the viscoelasticity of each joint is regulated using EMG signals.

In this chapter, the biomimetic control for multi-joint motion is explained in Section 2. Section 3 describes the proposed impedance control system, and Sections 4 and 5 describe experiments conducted to assess the effectiveness of the method. Finally, Section 6 concludes the chapter and discusses the research work in further detail.

\section{Biomimetic control of multi-joint motion}

\subsection{Control strategy}

Various types of human motion are generally realized by multiple skeletal muscles related to each joint. Accordingly, multi-joint motion can be controlled if muscular contraction levels related to all joints can be estimated accurately from EMG signals. Even if only the human wrist joint is considered, however, there are at least three degrees of freedom and more than ten muscles involved with complex interrelations. Moreover, EMG signals measured on the skin surface contain only information on surface muscles in the human body, and such signals have non-linear and non-stationary characteristics. Estimation of all forces and torques caused by the muscles is therefore extremely difficult.

To overcome these difficulties, this chapter introduces a new control method involving two steps: In the first, the operator's intended joint motion is estimated using surface EMG signals measured from the operator's skin, and the joints to be driven in the prosthetic hand are selected. In the second step, the muscular contraction levels are estimated, and the joints selected in the first step are controlled using the impedance control.

Although many studies on discrimination of motion from EMG patterns [7], [10], [11] and impedance control of a single-joint prosthetic arm using EMG signals [6], [8], [9] have been reported so far, no previous method has realized multi-joint motion control based on EMG pattern discrimination and impedance control using internal and external forces arising from agonist and antagonist muscles. In this chapter, the method proposed by Tsuji et al. [17] is used for the first step.

\subsection{Single-joint model considering flexors and extensors}

Skilful motion of the human arm is realized by regulating its impedance properties, such as stiffness, viscosity and inertia [13]. A natural feeling of control similar to that of the human arm can be expected if prosthetic control is performed on the basis of impedance control with human-arm impedance properties.

As shown in Fig. 1 (a), the characteristics of joint motion can be represented by the tension balance between flexors and extensors. Here, each muscle tension $f_{i}$ is modeled as

$$
f_{i}=f_{0 i}\left(\alpha_{i}\right)-k_{i}\left(\alpha_{i}\right) x_{i}-b_{i}\left(\alpha_{i}\right) \dot{x}_{i},
$$

where $\alpha_{i}\left(0 \leq \alpha_{i} \leq 1\right)$ is the muscular contraction level, $f_{0 i}\left(\alpha_{i}\right)$ is the muscle tension under isometric contraction at a natural length, and $x_{i}, k_{i}\left(\alpha_{i}\right)$ and $b_{i}\left(\alpha_{i}\right)$ are the displacement, muscular stiffness and muscular viscosity, respectively. The subscript $i \in\{f, e\}$ indicates flexor or extensor. 


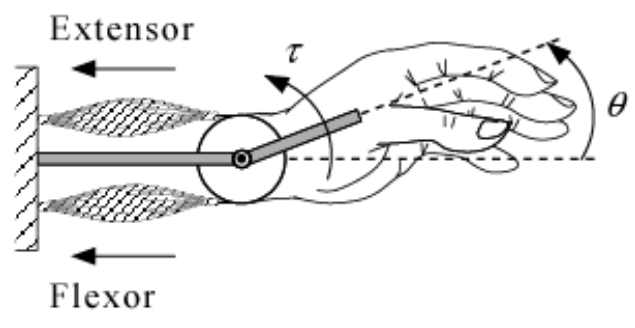

(a) Link model

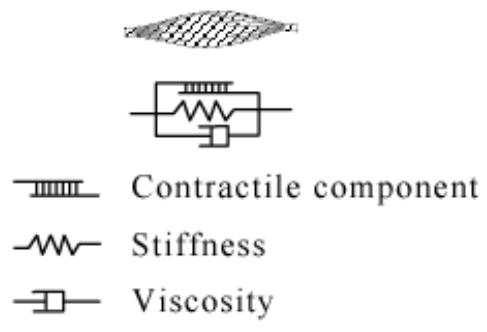

(b) Muscle model

Fig. 1. Musculoskeletal model of human wrist joint

Accordingly, the joint torques $\tau_{f}$ and $\tau_{e}$ generated by the flexor and extensor are also described as

$$
\tau_{i}=\tau_{0 i}\left(\alpha_{i}\right)-K_{i}\left(\alpha_{i}\right) \theta-B_{i}\left(\alpha_{i}\right) \dot{\theta}
$$

where $\tau_{0 i}\left(\alpha_{i}\right), K_{i}\left(\alpha_{i}\right)$ and $B_{i}\left(\alpha_{i}\right)$ are the joint torque at the natural muscle length, joint stiffness and joint viscosity, respectively, and $\theta$ is the joint angle. Thus, the equation for wrist joint motion is represented as

$$
\begin{aligned}
I & =\tau_{f}+\tau_{e} \\
& =\tau_{0}\left(\alpha_{f}, \alpha_{e}\right)-K\left(\alpha_{f}, \alpha_{e}\right) \theta-B\left(\alpha_{f}, \alpha_{e}\right) \dot{\theta}
\end{aligned}
$$

where $I, K\left(\alpha_{f}, \alpha_{e}\right)$ and $B\left(\alpha_{f}, \alpha_{e}\right)$ represent the impedance parameters of the wrist joint such as the moment of inertia, joint stiffness and viscosity, and $\tau_{0}\left(\alpha_{f}, \alpha_{e}\right)$ is the joint torque at the natural muscle length. In order to control the movement of the prosthetic hand based on (3), three problems must be solved: i) how to calculate muscle contraction levels $\alpha_{f}$ and $\alpha_{e}$ of the flexors and extensors during movement; ii) how to define the isometric joint torque $\tau_{0}\left(\alpha_{f}, \alpha_{e}\right)$; and iii) how to determine the impedance parameters of $I, K\left(\alpha_{f}, \alpha_{e}\right)$ and $B\left(\alpha_{f}, \alpha_{e}\right)$.

In this study, EMG signals and a neural network were utilized for the first and second problems. For the third one, the impedance parameters of wrist joints in non-amputee subjects were measured experimentally.

\section{Biomimetic impedance control of a robotic hand}

Figure 2 shows the proposed biomimetic impedance control system for a robotic hand. This system consists of four parts: force extraction; determination of the driven joint (raw EMG pattern discrimination); impedance control; and a robotic hand. The details of each part are described in following subsections. 


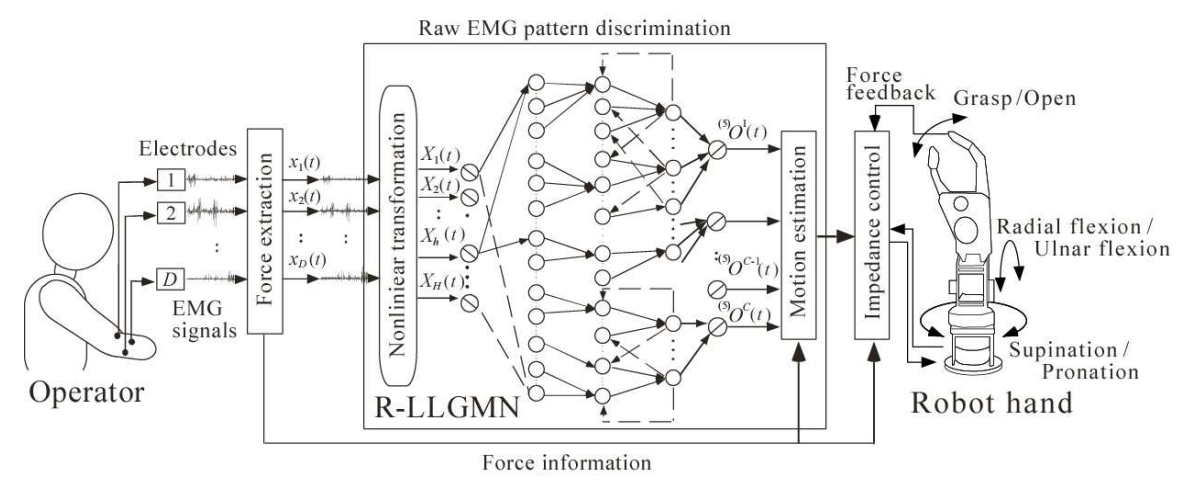

Fig. 2. Biomimetic control system for a robot hand

\subsection{Force extraction}

First, force information relating to the user was extracted from the EMG signals measured. The electrodes (NT-511G, NT-512G: Nihon Kohden Corp.) for EMG measurement were made of $\mathrm{Ag} / \mathrm{AgCl}$ with a diameter of $0.012 \mathrm{~m}$. The distance between the electrodes was set as $0.03 \mathrm{~m}$. The measured EMG signals were amplified and filtered out with a low-pass filter (cut-off frequency: $100 \mathrm{~Hz}$ ) in a multi-telemeter (Web5000: Nihon Kohden Corp.) and digitized using an A/D converter (sampling frequency: $200 \mathrm{~Hz}$; quantization: 12 bits) after amplification $(70 \mathrm{~dB})$. The $D$ channel EMG signals were denoted using $E_{d}(n)(d=1,2, \ldots, D ; n$ $=1,2, \ldots, N)$, where $N$ is the number of all data.

The integral EMG (IEMG) $\beta(n)$ were obtained by calculating the moving average within the teacher vector length $T$ of the neural network after rectification of $E_{d}(n)$ :

$$
\begin{array}{r}
\beta(n)=\frac{1}{D} \sum_{d=1}^{D} \frac{\overline{E M G}_{d}(n)}{\overline{E M G}_{d}^{\max }}, \\
\overline{E M G}_{d}(n)=\frac{1}{T} \sum_{t^{\prime}=0}^{T-1}\left|E_{d}\left(n-t^{\prime}\right)\right|,
\end{array}
$$

where $\overline{E M G}_{d}^{\text {max }}$ is the pre-measured IEMG of each channel under the maximum voluntary contraction (MVC). It should be noted that $E_{d}\left(n-t^{\prime}\right)=0$ when $n-t^{\prime}<0$. In this system, $\beta(n)$ is used for recognition of the beginning and end of motion (i.e., when $\beta(n)$ is above the motion appearance threshold $\beta_{d}$, motion is judged to have occurred).

The input vector $\mathbf{x}_{d}(t)=\left[x_{1}(t), x_{2}(t), \cdots, x_{d}(t)\right]^{\mathrm{T}}(t=1, \ldots, T)$ for the neural network is defined as the normalized $E_{d}(n)$ with $\beta(n)$ :

$$
x_{d}(t)=\beta^{-1}(T) E_{d}(t) .
$$

This normalization enables motion estimation from the pattern of all channels and the amplitude of the raw EMG signals. 
Furthermore, to allow impedance control of a robotic hand, the following values computed after $E_{d}(n)$ were rectified and filtered using a digital Butterworth filter (cut-off frequency: $f_{c}$ $\mathrm{Hz})$ :

$$
\begin{array}{r}
E_{\mu}(n)=\frac{1}{D} \sum_{d=1}^{D}\left(E_{d}(n)-E_{d}^{s t}\right), \\
\delta_{k}(n)=\frac{E_{\mu}(n)-E^{t h}}{E_{k}^{\text {max }}-E^{t h}},
\end{array}
$$

where $E_{d}^{s t}$ is the average of EMG signals $E_{d}(n)$ in a relaxed state and $E_{k}^{\max }$ is the premeasured $E_{\mu}(n)$ of each motion under the maximum voluntary contraction (MVC). $\delta_{k}(n)$ therefore describes the muscular contraction level for each motion $\left(0 \leq \delta_{k}(n)<1\right)$.

\subsection{Determination of the driven joint}

For the estimation of operator's intended motion, this subsection focuses on the pattern discrimination of the EMG signals using a probabilistic neural network (PNN). Since the PNN integrates statistical models into the neural network architecture as prior knowledge, outstanding performance has been reported [14]. For EMG pattern recognition using PNNs, the feature characteristics usually include: (1) amplitude, (2) frequency and (3) spatial information from multiple channels of EMG signals. However, significant temporal characteristics exist in the transient and non-stationary EMG signals, which cannot be considered by the traditional PNNs based on static stochastic models, and, in some cases, temporal characteristics could be only clues for reliable recognition. To overcome this problem, a Recurrent Log-Linearized Gaussian Mixture Network (R-LLGMN)[15] is utilized as the PNN for EMG pattern discrimination in the proposed system.

Since this network is composed of a feedforward NN including a Gaussian mixture model and feedback connections from output to input, the filtering process and the pattern discrimination are unified together and realized in a single network. The R-LLGMN includes a hidden Markov Model (HMM) [16] in its structure and can regulate the weight coefficients based on the learning scheme of the back-propagation through time (BPTT) algorithm [17]. The R-LLGMN ensures the filtering process and the pattern discrimination to be achieved at the same time and can attain high discrimination ability. The network therefore can classify time series of raw EMG signals [17].

The structure of R-LLGMN is shown in Fig. 2. This network is a five-layer recurrent NN with feedback connections between the 3rd layer and the 4th layer. First of all, the input vector $\mathbf{x}_{d}(t)=\left[x_{1}(t), x_{2}(t), \cdots, x_{d}(t)\right]^{\mathrm{T}} \in \mathfrak{R}^{d}$ is pre-processed with a non-linear computation and converted into the modified vector $\mathbf{X} \in \mathfrak{R}^{H}$ :

$$
\mathbf{X}(t)=\left[1, \mathbf{x}(t)^{\mathrm{T}}, x_{1}(t)^{2}, x_{1}(t) x_{2}(t), \ldots, x_{1}(t) x_{d}(t), x_{2}(t)^{2}, x_{2}(t) x_{3}(t), \ldots, x_{2}(t) x_{d}(t), \ldots, x_{d}(t)^{2}\right]^{\mathrm{T}} .
$$


Fig. 3. Structure of the R-LLGMN

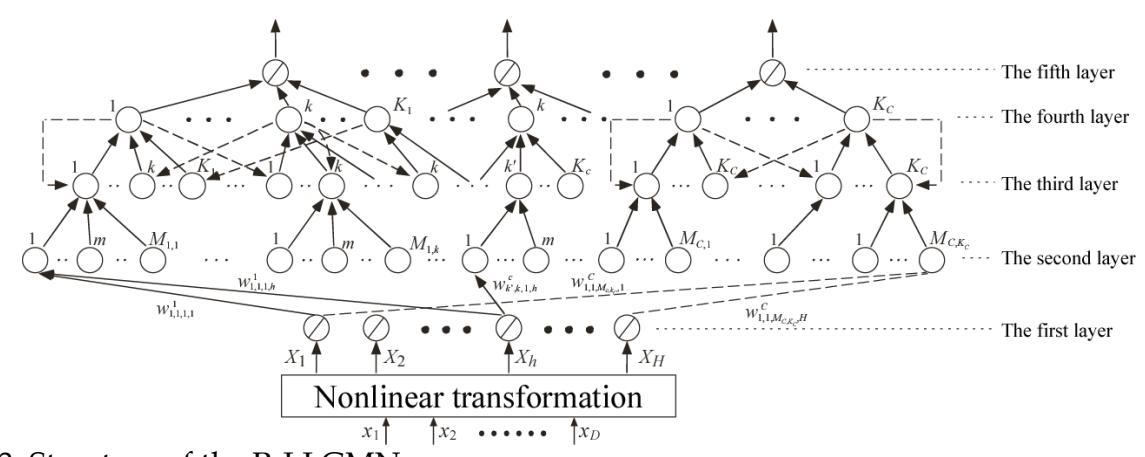

The first layer consists of $H$ units corresponding to the dimension of $\boldsymbol{X}$ (the dimension $H$ is determined as $H=1+d(d+3) / 2$ ) and the identity function is used for activation of each unit. Each unit in the first layer is defined as

$$
{ }^{(1)} I_{h}(t)=X_{h}(t), \quad{ }^{(1)} O_{h}(t)={ }^{(1)} I_{h}(t) ，
$$

where ${ }^{(1)} I_{h}(t)$ and ${ }^{(1)} O_{h}(t)$ denote the input and the output, respectively, of the $h$ th unit in the first layer.

Unit $\left\{c, k, k^{\prime}, m\right\}\left(c=1, \ldots, C ; k, k^{\prime}=1, \ldots K_{C} ; m=1, \ldots, M_{c, k}\right)$ in the second layer receives the output of the first layer weighted by the coefficient $w_{k^{\prime}, k, m, h}^{c}$. The relationship between the input and the output in the second layer is defined as

$$
\begin{gathered}
{ }^{(2)} I_{k^{\prime}, k, m}^{c}(t)=\sum_{h=1}^{H}{ }^{(1)} O_{h}(t) w_{k^{\prime}, k, m, h}^{c}, \\
{ }^{(2)} O_{k^{\prime}, k, m}^{c}(t)=\exp \left({ }^{(2)} I_{k^{\prime}, k, m}^{c}(t)\right),
\end{gathered}
$$

where $C$ is the number of classes, $K_{c}$ is the number of states, $M_{c, k}$ is the number of the components of the Gaussian mixture distribution corresponding to the class $c$ and the state $k$ [18].

The input into a unit $\left\{c, k, k^{\prime}\right\}$ in the third layer integrates the outputs of units $\left\{c, k, k^{\prime}, m\right\}$ $\left(m=1, \ldots, M_{c, k}\right)$ in the second layer. The output in the third layer is that input weighted by the previous output in the fourth layer. The input-output relationship of the unit in the third layer is defined as

$$
\begin{array}{r}
{ }^{(3)} I_{k^{\prime}, k}^{c}(t)=\sum_{m=1}^{M_{c, k}}{ }^{(2)} O_{k^{\prime}, k, m}^{c}(t), \\
{ }^{(3)} O_{k^{\prime}, k}^{c}(t)={ }^{(4)} O_{k^{\prime}}^{c}(t-1)^{(3)} I_{k^{\prime}, k}^{c}(t),
\end{array}
$$


where ${ }^{(4)} O_{k^{\prime}}^{c}(0)=1.0$ for the initial state.

The fourth layer receives the integrated outputs of units $\left\{c, k, k^{\prime}\right\}$ in the third layer. The input-output relationship in the fourth layer is defined as

$$
\begin{array}{r}
{ }^{(4)} I_{k}^{c}(t)=\sum_{k^{\prime}=1}^{K_{c}}{ }^{(3)} O_{k^{\prime}, k}^{c}(t), \\
{ }^{(4)} O_{k}^{c}(t)=\frac{{ }^{(4)} I_{k}^{c}(t)}{\sum_{c^{\prime}=1}^{C} \sum_{k^{\prime}=1}^{K}{ }^{(4)} I_{k^{\prime}}^{c^{\prime}}(t)} .
\end{array}
$$

At last, a unit $c$ in the fifth layer integrates the outputs of $K_{c}$ units $\{c, k\}\left(k=1, \ldots K_{C}\right)$ in the fourth layer. The relationship in the fifth layer is defined as

$$
\begin{array}{r}
{ }^{(5)} I^{c}(t)=\sum_{k=1}^{K_{c}}{ }^{(4)} O_{k}^{c}(t), \\
{ }^{(5)} O^{c}(t)={ }^{(5)} I^{c}(t) .
\end{array}
$$

The output of the network ${ }^{(5)} O^{c}(t)$ corresponds to the a posteriori probability of the input vector $\mathbf{x}(t)$ for the class $c$, while only the weight coefficients $w_{k^{\prime}, k, m, h}^{c}$ between the first layer and the second layer are adjusted by learning.

The entropy of R-LLGMN's output is calculated to prevent the risk of misdiscrimination. The entropy is defined as

$$
H(t)=-\sum_{c=1}^{C}{ }^{(5)} O^{c}(t) \log _{2}{ }^{(5)} O^{c}(t)
$$

If the entropy $H(t)$ is less than the discrimination threshold $H_{d}$, the specific motion with the largest probability is determined according to the Bayes decision rule. If not, the determination is suspended.

The details of the raw EMG pattern discrimination using the R-LLGMN are described in [17]. In the proposed system, the driven joint $j$ is selected based on the output of the R-LLGMN. 


\subsection{Impedance control}

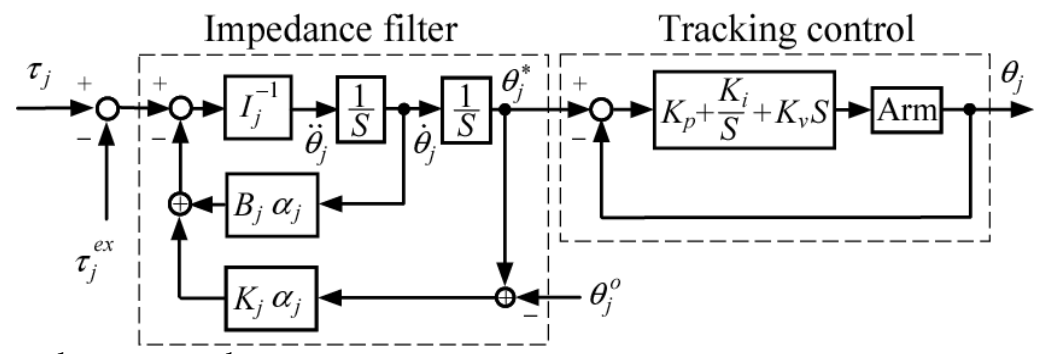

Fig. 4. Impedance control system

Human hand and wrist movement can be expressed based on a mechanical impedance model encompassing stiffness, viscosity and inertia [13]. The introduction of a human hand and wrist impedance model to control a robotic hand will make it possible to realize a natural feeling of control similar to that of human movement. This subsection explains a method to control a robot hand based on the mechanical impedance of the human hand's wrist movements.

First, the equation of motion around the manipulator's joint $j$ is defined as

$$
I_{j} \ddot{\theta}_{j}+B_{j}\left(\alpha_{j}\right) \dot{\theta}_{j}+K_{j}\left(\alpha_{j}\right)\left(\theta_{j}-\theta_{j}^{o}\right)=\tau_{j}-\tau_{j}^{e x},
$$

where $I_{j}, B_{j}\left(\alpha_{j}\right)$ and $K_{j}\left(\alpha_{j}\right)$ are the moment inertia, joint viscosity and stiffness, respectively. Here, it is assumed that the joint torques caused by muscular contraction of the flexors and extensors have almost the same properties, and the muscular contraction level of each joint is expressed as $\alpha_{j}=\alpha_{j, f}+\alpha_{j, e} . \theta_{j}$ and $\theta_{j}^{o}$ describe the joint angle and its equilibrium position, and $\tau_{j}$ and $\tau_{j}^{e x}$ are the joint torque and external torque, respectively. Muscular contraction level $\alpha_{j}$ and joint torque $\tau_{j}$ can be expressed as follows:

$$
\begin{gathered}
\alpha_{j}=\delta_{k}(n), \\
\tau_{j}(n)=\delta_{k}(n) \tau_{k}^{\max },
\end{gathered}
$$

where $j$ is the joint driven during motion $k$, and $\tau_{k}^{\max }$ describes the maximum torque measured from the subject for each motion $k$ in advance. It should be noted that the torque is assumed to remain the same (i.e., $\left.\tau_{j}(n)=\tau_{j}(n-1)\right)$ when the discrimination results are suspended.

Using the above equations, the desired joint angles can be calculated numerically using dynamic equation (20) considering changes in the EMG signals. Figure 4 shows the impedance control system used in this study. Here, $K_{p}, K_{i}$ and $K_{v}$ are the gain parameters for PID control. This method can be expected to provide a natural feeling of control similar to that of the original limb if the impedance parameters are set as values similar to those of the human arm. The hand can also react to external forces using a force sensor. 


\subsection{Robotic hand [12]}
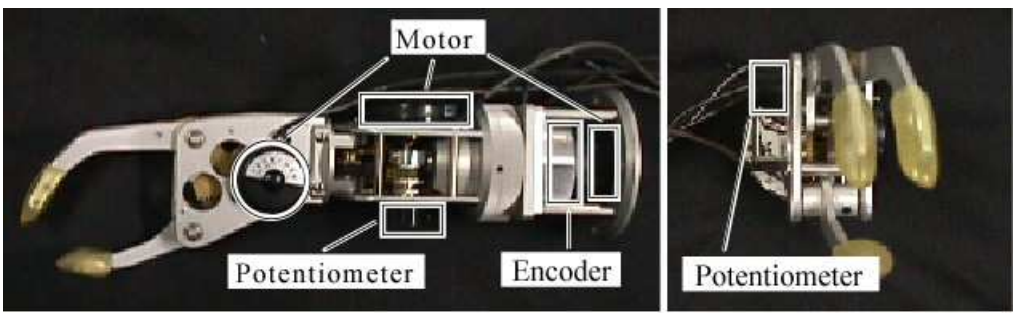

Fig. 5. Robotic hand

A photograph of the robotic hand utilized in this study is shown in Fig. 5 [12]. It is almost the same size as an adult hand, and weighs about $1.0[\mathrm{~kg}]$. The hand has three degrees of freedom (supination/pronation, radial flexion/ulnar flexion, grasp/open), and each joint is driven by an ultrasonic motor (Sinsei Corp.) [7]. An encoder or potentiometer is installed as an angular sensor for each joint as shown in Fig. 5. The unit can be attached to the amputation site and used as a prosthetic hand.

\section{Determination of human wrist impedance parameters}

Impedance control is an effective technique for achieving natural feeling similar to that of a human hand in prosthetic control. However, it is difficult to set the relevant parameters appropriately. Measurement experiments to ascertain human wrist joint impedance characteristics were therefore carried out.

\subsection{Measurement of human wrist joint impedance}

This section outlines Tsuji's method [13] for measurement of human wrist joint impedance. In a case where the subject performs single-joint motion of the wrist on a $2 \mathrm{D}$ plane, the dynamic properties of the hand can be approximated using a mechanical impedance model on a $2 \mathrm{D}$ plane as follows:

$$
I_{h} \ddot{\theta}(t)+B_{h} \dot{\theta}(t)+K_{h} \theta(t)=\tau(t),
$$

where $\theta(t)$ is the joint angle, $\tau(t)$ is the joint torque, and $I_{h}, B_{h}$ and $K_{h}$ are the moment of inertia, joint viscosity and stiffness, respectively.

In order to estimate the relevant parameters, the hand of the subject is displaced from equilibrium by means of a small short-duration disturbance (Fig. 6). A small disturbance is necessary to enable the assumption of an approximate constancy for $I_{h}, B_{h}$ and $K_{h}$, which are known to depend on posture in smooth conditions. Here, at the onset time $t_{0}$ of the disturbance, we have

$$
I_{h} \ddot{\theta}\left(t_{0}\right)+B_{h} \dot{\theta}\left(t_{0}\right)+K_{h} \theta\left(t_{0}\right)=\tau\left(t_{0}\right) .
$$

The dynamic hand properties at given time $t$ are established by Eqs. (23) and (24): 


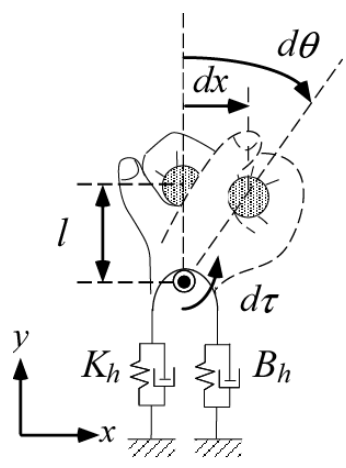

Fig. 6. Impedance model of the wrist joint

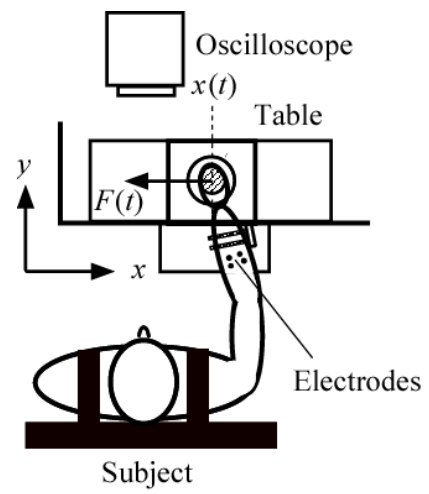

Fig. 7. Experimental apparatus

$$
I_{h} d \ddot{\theta}(t)+B_{h} d \dot{\theta}(t)+K_{h} d \theta(t)=d \tau(t),
$$

where $d \theta(t)=\theta(t)-\theta\left(t_{0}\right)$ and $d \tau(t)=\tau(t)-\tau\left(t_{0}\right)$. The parameters $I_{h}, B_{h}$ and $K_{h}$ of the subject can be estimated using the least square method from $n$ equations for each sample $\left(t=t_{1}, \ldots, t_{n}\right)$. Figure 7 shows the experimental apparatus for measurement of impedance parameters, which uses a linear motor table (Nippon Thompson Co., Ltd.) with one degree of freedom adopting a magnetic drive to forcibly displace the subject's hand. In the experiments, the right hand of the subject sitting in front of the table was fixed to a handle through a cast made of glass fiber. In addition, the subject's forearm was fixed to an arm-supporting stand by the cuff. The surface EMG was then measured from the agonist and antagonist muscles of the wrist joint in the subject's forearm in order to clarify the activity of the muscles around the joint. A display for online monitoring of the muscle contraction level was set in front of the subject. The task for the subjects involved isometric flexion and extension movement of the wrist joint. During the experiments, the subjects could regulate the muscle contraction level as instructed by the experimenter since the contraction levels of each 
muscle were monitored by the display. The force generated by the subject was measured using a six-axis force sensor (BL Autotec Co. Ltd; resolution ability: force $x$-and $y$-axes: 0.05 $\mathrm{N}$; z-axis: $0.15 \mathrm{~N}$ ) attached to the handle of the table. The output from the encoder and force sensor were sampled at $2 \mathrm{kHz}$.

From the slight hand displacement in the $x$ axial direction $x(t)$ and the hand's resultant force along the $x$ axis $F_{x}(t)$, we obtain the joint angle displacement $\theta(t)$ and joint torque $\tau(t)$ using the following equations:

$$
\begin{aligned}
& \theta(t)=\sin ^{-1}(x(t) / l), \\
& \tau(t)=l F_{x}(t),
\end{aligned}
$$

where $l$ is the distance from the handle to the center of the wrist joint (see Fig. 6).

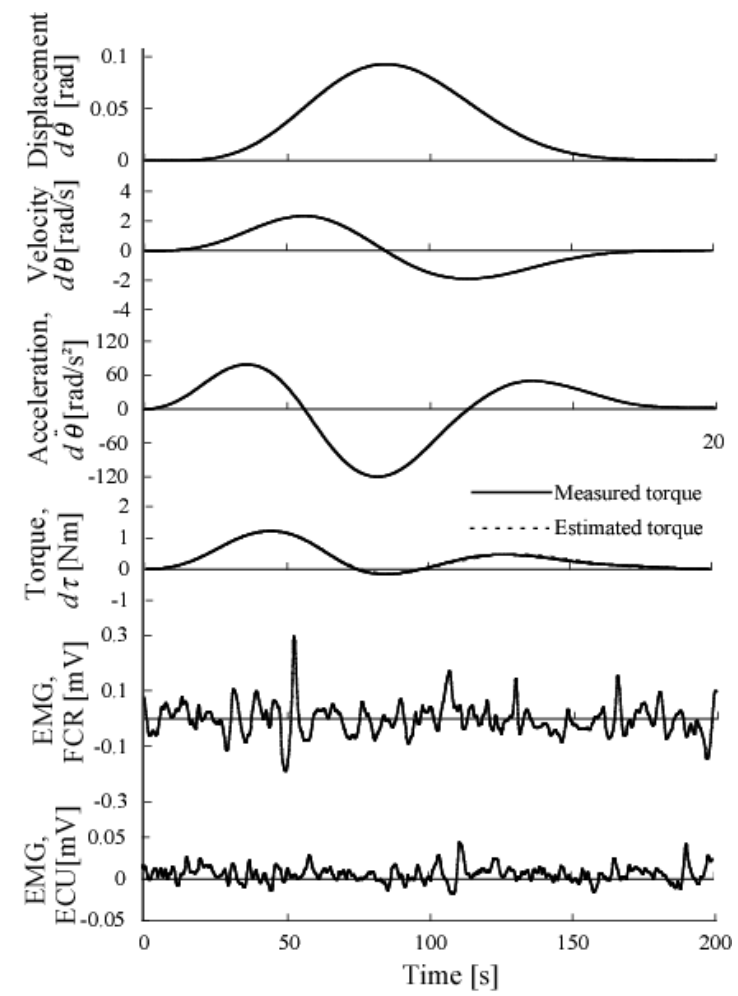

Fig. 8. A typical example of measured results for human wrist while maintaining posture

\subsection{Results}

Four healthy male subjects participated in the experiments. Figure 8 shows an example of the measurement results for wrist joint displacement in the extension direction. The figure shows the joint angle $d \theta(t)$, velocity $d \dot{\theta}(t)$, acceleration $d \ddot{\theta}(t)$, wrist joint torque $d \tau(t)$ and the EMG signals of the flexor carpi radialis (FCR) and extensor carpi ulnaris (ECU). From 
the figure, it can be seen that the measured torque (shown by the solid line) corresponds with the estimated torque (the dotted line) using the measured $d \theta(t), d \dot{\theta}(t)$ and $d \ddot{\theta}(t)$ and the impedance parameters $I_{h}, B_{h}$ and $K_{h}$ computed from them. The results indicate that an impedance model based on Eqs. (23), (24) and (25) can express the human wrist joint's characteristics.

Examples of the relationships between the muscular contraction levels and estimated parameters $I_{h}, B_{h}$ and $K_{h}$ are shown in Fig. 9. In this figure, $\alpha(0 \leq \alpha \leq 1)$ indicates the muscular contraction levels estimated from the EMG signals, and the plotted data show the measured values. From the figure, moment inertia $I_{h}$ is maintained at a constant value, and viscosity $B_{h}$ and stiffness $K_{h}$ change according to the level of muscle contraction. In particular, $B_{h}$ and $K_{h}$ increase as muscle contraction levels become high. This study therefore approximated the relationships between muscular contraction levels and the impedance properties of stiffness and viscosity around the wrist joint as follows:

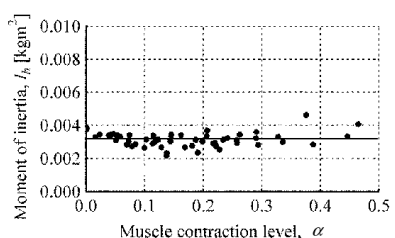

(a) Estimated moment of inertia of Sub. A

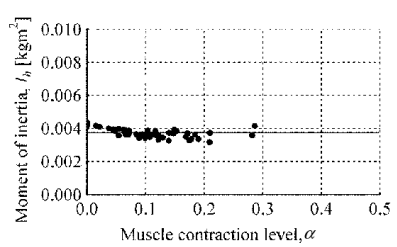

(d) Estimated moment of inertia of Sub. B

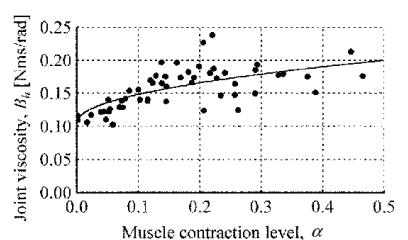

(b) Estimated joint viscosity of Sub. A

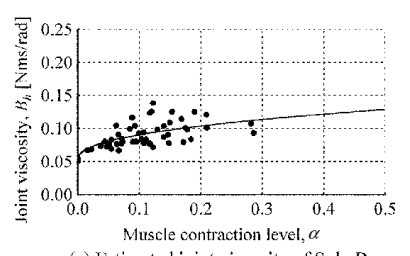

(e) Estimated joint viscosity of Sub. B

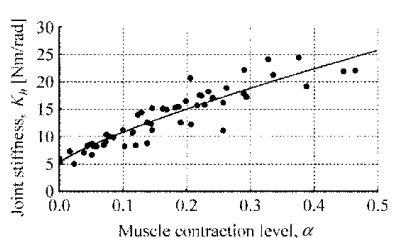

(c) Estimated joint stiffeness of Sub. A

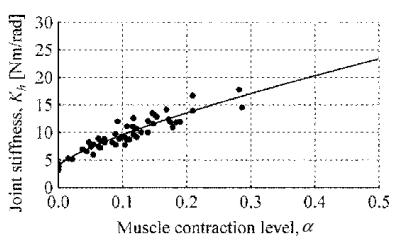

(f) Estimated joint stiffness of Sub. $B$

Fig. 9. Estimated impedance parameters of human wrist joint

$$
\begin{aligned}
& \hat{B}_{h}(\alpha)=b_{1} \alpha^{b_{2}}+b_{3}, \\
& \hat{K}_{h}(\alpha)=k_{1} \alpha^{k_{2}}+k_{3},
\end{aligned}
$$

where $\hat{B}_{h}(\alpha)$ and $\hat{K}_{h}(\alpha)$ are the estimated values of viscosity and stiffness around the wrist joint, $k_{i}$ and $b_{i}(i=1,2,3)$ are constants, and $k_{3}$ and $b_{3}$ correspond to the level of viscoelasticity seen when the arm is in a relaxed state. The solid line in Fig. 9 shows the impedances estimated by Eqs. (28) and (29) using the least square method from the values obtained. From the figure, it is seen that the tendency of each parameter can be expressed using Eqs. (28) and (29). 


\section{Robotic hand control experiments}

\subsection{Experimental conditions}

In the experiments, the cut-off frequency $f_{c}$ and sampling frequency $f_{s}$ were set as $3.0 \mathrm{~Hz}$ and $1,000 \mathrm{~Hz}$, respectively. Based on the above-mentioned experiments, the impedance parameters for controlling the robotic hand were set as follows:

$$
\begin{gathered}
I_{j}=i_{j,} \\
B_{j}\left(\alpha_{j}\right)=b_{j, 1} \alpha_{j}^{b_{j, 2}}+b_{j, 3} \\
K_{j}\left(\alpha_{j}\right)=k_{j, 1} \alpha_{j}^{k_{j, 2}}+k_{j, 3},
\end{gathered}
$$

where $j=1,2,3$ is the joint number, and each parameter in Eqs. (30), (31) and (32) is shown in Table 1. Four healthy male subjects participated in the experiments. It should be noted that the impedance parameters estimated from the human impedance characteristics measured were for the wrist joint only (i.e., flexion and extension), and the parameters of other joints were defined by trial and error based on the wrist joint characteristics.

\begin{tabular}{c|c||c|c|c||c|c|c|c}
\hline \hline joint $j$ & Motion (k) & $k_{j, 1}$ & $k_{j, 2}$ & $k_{j, 3}$ & $b_{j, 1}$ & $b_{j, 2}$ & $b_{j, 3}$ & $i_{j}$ \\
\hline 1 & Pronation (1)/ spination (2) & 32.0 & 0.6 & 3.2 & 0.14 & 0.2 & 0.110 & 0.002 \\
\hline 2 & Ulnar flexion (3) / radial flexion (4) & 32.8 & 0.6 & 3.2 & 0.14 & 0.2 & 0.144 & 0.004 \\
\hline 3 & Open (5)/ close (6) & 0.90 & 0.6 & 0.3 & 0.08 & 0.2 & 0.090 & 0.001 \\
\hline \hline
\end{tabular}

Table 1. Impedance parameters used in the experiments

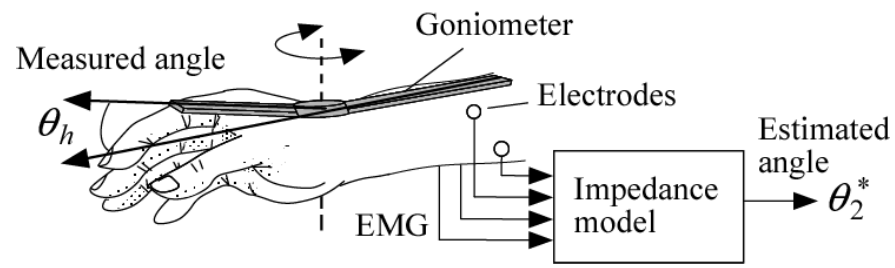

Fig. 10. Estimation of the joint angle from EMG signals based on biomimetic impedance model

\subsection{Biomimetic impedance control}

Here, in order to examine the validity of the impedance parameters, the motion of the subject's wrist joint and that of the manipulator were compared.

The subject executed wrist flexion and extension, and the wrist joint angle and EMG signals were measured as shown in Fig. 9. The muscle contraction level $\alpha_{j}$ was calculated from the EMG signals using the proposed method, and the desired joint angle $\theta_{j}^{*}$ (see Fig. 10) was calculated according to the muscle contraction level and the motion equation for the wrist joint with variable viscoelasticity (Eqs. (20), (21), and (22)). 


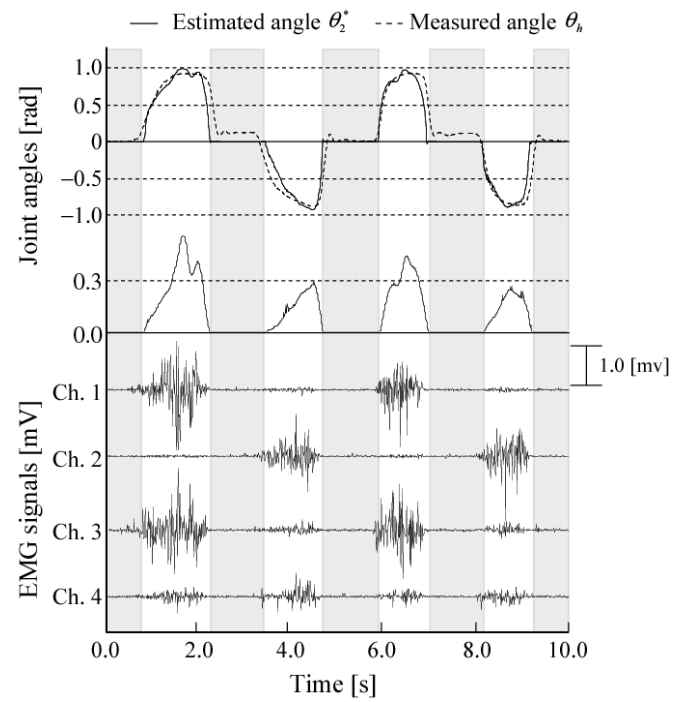

Fig. 11. Comparison between the estimated and measured angles of the wrist joint

In the experiment, four electrodes $(L=4: 1 \mathrm{ch}$. flexor carpi radialis, FCR; $2 \mathrm{ch}$. flexor carpi ulnaris, FCU; 3 ch. extensor carpi radialis, ECR; and $4 \mathrm{ch}$. brachioradialis, BR) were used, two motions ( $k=2$; flexion and extension) were discriminated using the neural network, and the motion appearance threshold was set as $E_{d}=0.17$.

Figure 11 shows an example of the experimental results. The wrist joint angle of the manipulator almost corresponds with that of the subject. Moreover, the maintenance of posture under conditions of muscle co-contraction was realized. It can be seen that the joint angles of the prosthetic hand can be controlled using EMG signals.

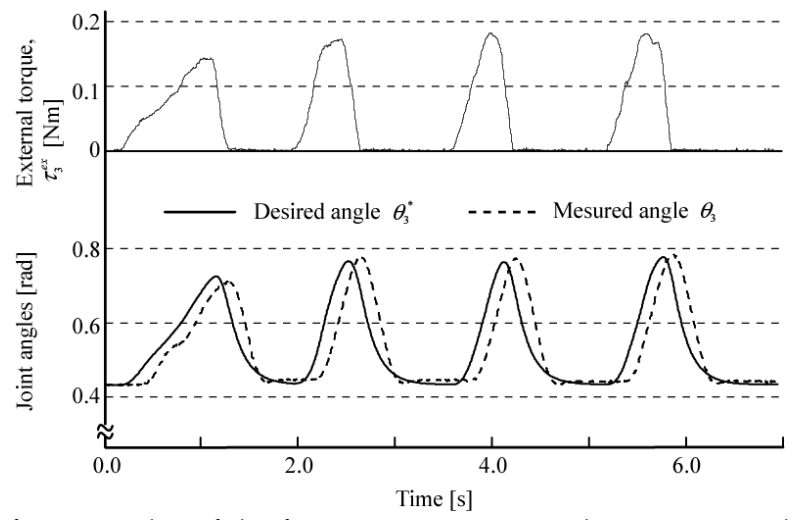

Fig. 12. Changes of joint angles of the finger part corresponding to external torque 


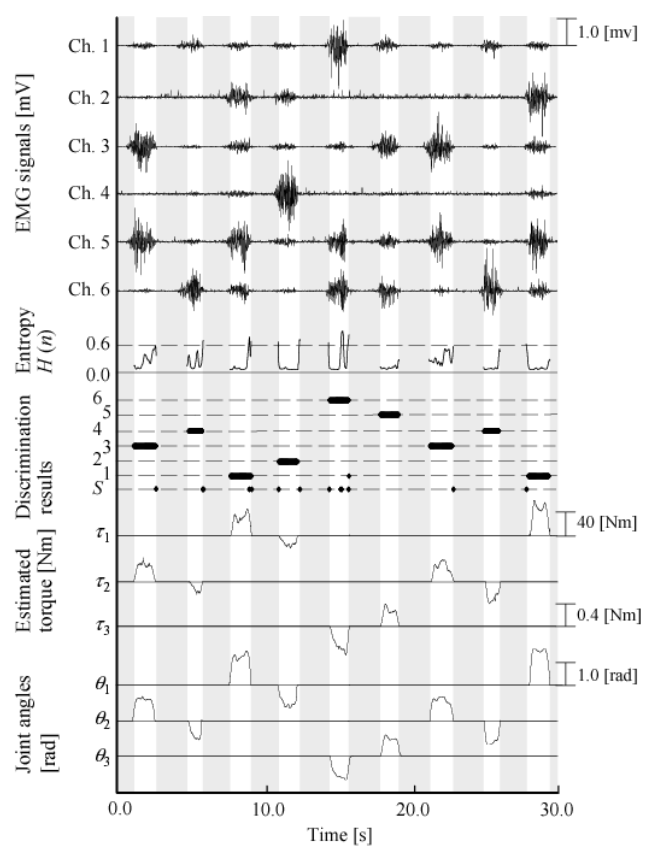

Fig. 13. An example of experimental control results (healthy subject)

To verify the response characteristics of the robotic hand for external torque, its movement was investigated by a human pressing the distal parts of its finger (attached to a pressure sensor) during in a relaxed state $\left(\alpha_{j}=0\right)$. The experimental results are shown in Fig. 12, in which the solid line shows the desired joint angle $\theta_{3}^{*}$ computed based on the impedance model, and the dotted line shows the joint angle $\theta_{3}$ obtained (see Fig. 4). From the figure, it can be observed that the joint angle of the robotic hand changes according to the external force applied, but a time delay between the measured and estimated values is seen. This was mainly caused by the control gains of the PID controller being set low $\left(K_{p}=0.16, K_{i}=0.001\right.$ and $\left.K_{v}=0.006\right)$ because the robotic hand, which is operated with ultrasonic motors, cannot provide smooth control with large gains.

\subsection{Robotic hand control}

Control experiments of the robotic hand based on EMG signals were carried out to verify the proposed method using the estimated muscular contraction levels. The experimental conditions were almost the same as those of the previous experiments. Six electrodes $(L=6: 1 \mathrm{ch}$. flexor carpi radialis; $2 \mathrm{ch}$. triceps brachii; $3 \mathrm{ch}$. extensor carpi radialis; $4 \mathrm{ch}$. biceps brachii; $5 \mathrm{ch}$. brachioradialis; and $6 \mathrm{ch}$. flexor carpi ulnaris) were attached to the forearm of a healthy subject (male, 25 years old), and EMG signals were measured. Figure 13 shows an example of the experimental results, including EMG signals from the six channels, entropy, discrimination results, estimated torque and joint angle; the shaded areas indicate periods with no motion. From the figure, it can be seen that the measured EMG signals can be classified accurately using the proposed method, and that the joint angles of the robotic hand can be controlled using these EMG signals. 


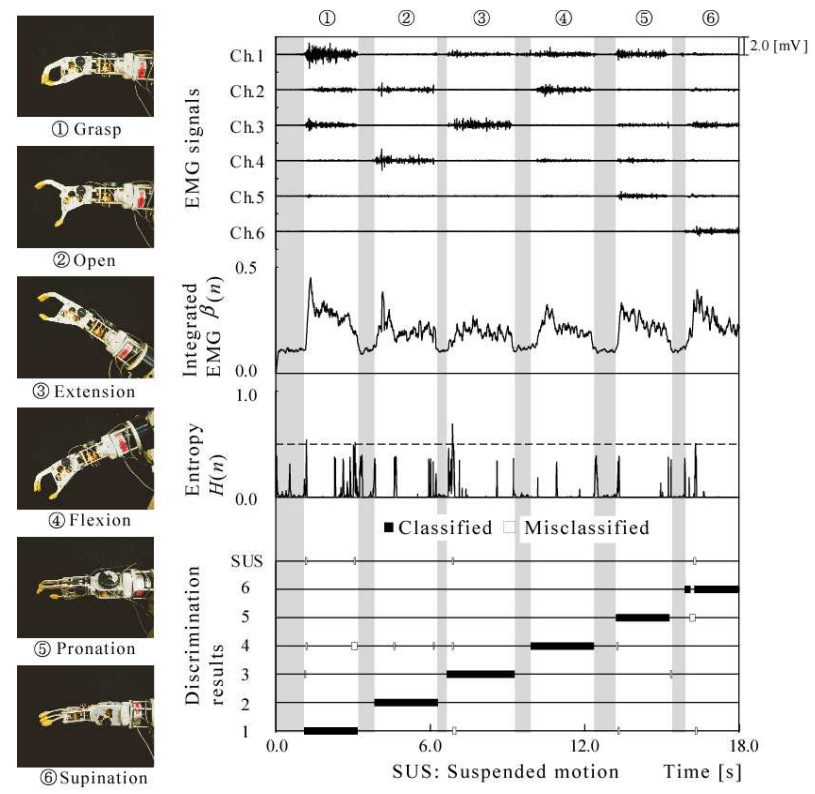

Fig. 14. Changes in the joint angles of the finger part in response to external torque

To confirm the suitability of the proposed method for people with physical disabilities, the EMG signals of an amputee subject (male, 44 years old) were measured and used to control the robotic hand for verification experiments. The subject's forearm was amputated at the age of 41. In this experiment, the R-LLGMN parameters were set as follows: the number of classes was $K=6$; the number of states for each class was $K_{1}, \ldots, K_{6}=1$; the number of components was $M_{1,1}, \ldots, M_{6,1}=1$; the teacher vector length was $T=20$ (about $100 \mathrm{~ms}$ ), and eight data sets were used for learning. Figure 14 shows the experimental results, including photographs of the robotic hand during control, raw EMG signals, integrated EMG (IEMG), entropy and discrimination results, respectively. The shaded area shows periods when no motion was judged from the IEMG. From the figure, it can be seen that the subject could control the robotic hand smoothly using EMG patterns, and the discrimination rate in this operation was $95.5 \%$. The results lead us to conclude that the proposed method can be used for robotic hand control based on human impedance characteristics.

\section{Conclusion}

This chapter explained a biomimetic impedance control method based on raw EMG classification for the development of a multi-joint robotic control system. In the experiments performed, a natural feeling of prosthetic control similar to that of the human hand was realized using the neural network and the biomimetic impedance control.

In the future, the authors plan to improve accuracy of estimation for the cooperation ratio of multiple muscles and construct a system that allows a more natural feeling of control.

Publications concerning this chapter are listed in the bibliography [17], [19]. 


\section{References}

[1] Wiener, N. (1948); CYBERNETICS or Control and Communication in the Animal and the Machine, MIT Press

[2] Sherman, E. D. (1964); A Russian Bioelectric-Controlled Prosthesis, Canada, M. A. J., 91, pp. $1268-1270$

[3] Kato, I.; Okazaki, E.; Kikuchi H.; Iwanami K. (1967); Electro-Pneumatically Controlled Hand Prosthesis Using Pattern Recognition of Myu-Electric Signals, Digest of 7 th ICMBE, p. 367

[4] Jerard, R. B.; Williams, T. W.; Ohlenbusch, C. W. (1974); Practical Design of an EMG Controlled Above Elbow Prosthesis, Proceedings of 1974 Conference on Engineering of Devices for Rehabilitation, Tufts Univ. School of Med., p. 73, Boston, MA

[5] Jacobson, S. C.; Knutti, D. F.; Johnson, R. T.; Sears, H. H. (1982); Development of the Utah Artificial Arm, IEEE Transactions on Biomedical Engineering, Vol. 29, No. 4, pp. 249269, April

[6] Akazawa, K.; Takizawa, H.; Hayashi, Y.; Fujii, K. (1988); Development of Control System and Myoelectric Signal Processor for Bio-Mimetic Prosthetic Hand, Biomechanism 9, pp. 43-53 (in Japanese)

[7] Ito, K.; Tsuji, T.; Kato, A.; Ito, M. (1992); An EMG Controlled Prosthetic Forearm in Three Degrees of Freedom Using Ultrasonic Motors, Proceedings of the Annual International Conference of the IEEE Engineering in Medicine and Biology Society, Vol. 14, pp. 14871488

[8] Abul-haj, C. J.; Hogan, N. (1990); Functional Assessment of Control Systems for Cybernetic Elbow Prostheses-Part I, Part II, IEEE Transactions on Biomedical Engineering, Vol. 37, No. 11, pp. 1025-1047, November

[9] Ito, K.; Tsuji, T. (1985); Control Properties of Human-Prosthesis System with Bilinear Variable Structure, Proceedings of the 2nd IFAC Conference on Man-Machine Systems, pp. 353-358

[10] Graupe, D. ; Magnussen, J.; Beex, A. A. M. (1978); A Microprocessor System for Multifunctional Control of Upper Limb Prostheses via Myoelectric Signal Identification, IEEE Transactions on Automatic Control\}, Vol. 23, No. 4, pp. 538-544, August

[11] Hiraiwa, A.; Shimohara, K.; Tokunaga, Y. (1989); EMG Pattern Analysis and Classification by Neural Network, Proceedings of IEEE International Conference on System, Man, and Cybernetics, pp. 1113-1115

[12] Fukuda, O.; Tsuji, T.; Kaneko, M.; Otsuka, A. (2003); A Human-Assisting Manipulator Teleoperated by EMG Signals and Arm Motions, IEEE Transactions on Robotics and Automation, Vol.19, No.2, pp.210-222, April

[13] Tsuji, T. (1997): Human arm impedance in multi-joint movements, Self-Organization, Computational Maps and Motor Control (P. Morasso and V. Sanguineti, Ed.), Advances in Psychology, 119, Elsevier, North-Holland, 1997, pp. 357-382

[14] Zhang, G.D. (2000); Neural network for classification: A survey, IEEE Transactions on Systems, Man and Cybernetics, Part C, Applications and Reviews, Vol. 30, pp. 451-462

[15] Tsuji, T.; Bu, N.; Fukuda, O.; Kaneko, M.; (2003); A Recurrent Log-linearized Gaussian Mixture Network, IEEE Transactions on Neural Networks, Vol. 14, No. 2, pp. 304-316, March 
[16] Rabiner, L.R. (1989); A tutorial on hidden Markov model and selected applications in speech recognition. Proceedings of the IEEE, vol. 77, pp. 257-286

[17] Tsuji, T.; Bu, N.; Fukuda, O. (2006); A Recurrent Probabilistic Neural Network for EMG Pattern Recognition, Neural Networks in Healthcare: Potential and Challenges (R. Begg, J. Kamruzzaman, R. Sarker, Ed.), pp. 130-153 (Idea Group Inc.)

[18] Tsuji, T.; Fukuda, O.; Ichinobe, H.; Kaneko, M. (1999); A Log-Linearized Gaussian Mixture Network and Its Application to EEG Pattern Classification, IEEE Transactions on Systems, Man, and Cybernetics-Part C: Applications and Reviews, Vol. 29, No. 1, pp. 60-72, February

[19] Tsuji, T.; Fukuda, O.; Shigeyoshi, H.; Kaneko, M. (2000); Bio-mimetic Impedance Control of an EMG-Controlled Prosthetic Hand, CD-ROM Proceedings of the 2000 IEEE/RSJ International Conference on Intelligent Robots and Systems, Kyongju 


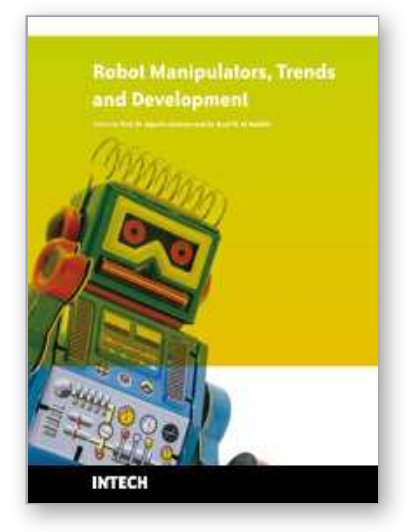

\author{
Robot Manipulators Trends and Development \\ Edited by Agustin Jimenez and Basil M Al Hadithi
}

ISBN 978-953-307-073-5

Hard cover, 666 pages

Publisher InTech

Published online 01, March, 2010

Published in print edition March, 2010

This book presents the most recent research advances in robot manipulators. It offers a complete survey to the kinematic and dynamic modelling, simulation, computer vision, software engineering, optimization and design of control algorithms applied for robotic systems. It is devoted for a large scale of applications, such as manufacturing, manipulation, medicine and automation. Several control methods are included such as optimal, adaptive, robust, force, fuzzy and neural network control strategies. The trajectory planning is discussed in details for point-to-point and path motions control. The results in obtained in this book are expected to be of great interest for researchers, engineers, scientists and students, in engineering studies and industrial sectors related to robot modelling, design, control, and application. The book also details theoretical, mathematical and practical requirements for mathematicians and control engineers. It surveys recent techniques in modelling, computer simulation and implementation of advanced and intelligent controllers.

\title{
How to reference
}

In order to correctly reference this scholarly work, feel free to copy and paste the following:

Toshio Tsuji, Keisuke Shima, Nan Bu and Osamu Fukuda (2010). Biomimetic Impedance Control of an EMGBased Robotic Hand, Robot Manipulators Trends and Development, Agustin Jimenez and Basil M Al Hadithi (Ed.), ISBN: 978-953-307-073-5, InTech, Available from: http://www.intechopen.com/books/robotmanipulators-trends-and-development/biomimetic-impedance-control-of-an-emg-based-robotic-hand

\section{INTECH}

open science | open minds

\author{
InTech Europe \\ University Campus STeP Ri \\ Slavka Krautzeka 83/A \\ 51000 Rijeka, Croatia \\ Phone: +385 (51) 770447 \\ Fax: +385 (51) 686166 \\ www.intechopen.com
}

\author{
InTech China \\ Unit 405, Office Block, Hotel Equatorial Shanghai \\ No.65, Yan An Road (West), Shanghai, 200040, China \\ 中国上海市延安西路65号上海国际贵都大饭店办公楼 405 单元 \\ Phone: +86-21-62489820 \\ Fax: $+86-21-62489821$
}


(C) 2010 The Author(s). Licensee IntechOpen. This chapter is distributed under the terms of the Creative Commons Attribution-NonCommercialShareAlike-3.0 License, which permits use, distribution and reproduction for non-commercial purposes, provided the original is properly cited and derivative works building on this content are distributed under the same license. 